Workshadowing in einer Londoner Kinderklinik

\title{
Fortgeschrittene Pflegepraxis
}

Die Schweizer Autorin Seraina Brooks befindet sich mitten im Studium zum Master of Science in Pflege, als sie beschließt, zum Workshadowing nach London zu gehen. Im Great Ormond Street Hospital for Children bekommt sie die Gelegenheit, den Alltag des APN-Teams mitzuerleben. Für uns beschreibt sie anhand verschiedener Praxisbeispiele die typischen Tätigkeiten einer Advanced Practice Nurse in der Onkologie und Hämatologie.

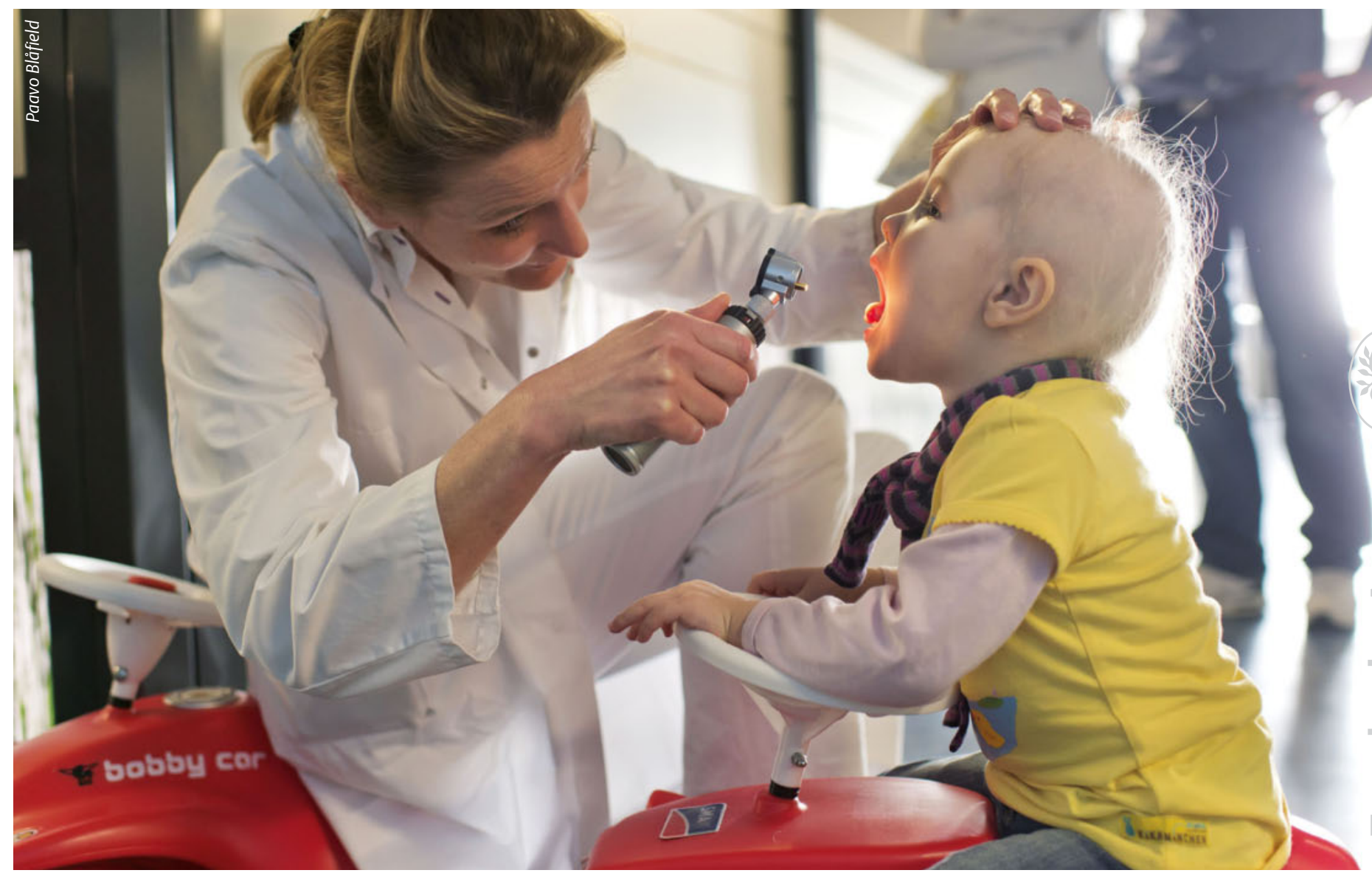

Während zwei Wochen begleitete ich im Rahmen meines WorkShadowings das APN-Team der pädiatrischen Onkologie und Hämatologie im stationären und ambulanten Bereich und erlebte den beruflichen Alltag der Clinical Nurse Specialist (CNS) wie auch der Nurse Practitioner (NP). Das Ziel dieses Einblicks-Praktikums war zu beobachten, wie die Familienzentrierung in der fortgeschrittenen Pflegepraxis implementiert ist und wie sich die interdisziplinäre Zusammenarbeit eines APN-Teams gestaltet. Letztlich war es mir wichtig, die unterschiedlichen Rollen von APNs in Großbritannien zu erleben.

Das Great Ormond Street Hospital wurde vor über 160 Jahren als eines der ersten Kinderspitäler im angelsächsischen Raum gegründet und gehört mit den über 50 pädiatrischen Disziplinen und Subdisziplinen zu den weltweit führenden Kinderkliniken. ${ }^{1}$ Das Departement für Onkolo- gie und Hämatologie gehört in Zusammenarbeit mit dem University College London zu den größten europäischen Krebszentren für Kinder. Fast ein Drittel der krebskranken Kinder in Großbritannien werden am Great Ormond Street Hospital behandelt. ${ }^{2}$

Die Familien auf den stationären und ambulanten Abteilungen für Onkologie und Hämatologie werden, neben dem Pflegeteam der jeweiligen Station, von einem APN-Team bestehend aus CNS und NP betreut. 


\section{Advanced Practice Nursing im Great Ormond Street Hospital für Kinder in London}

In Großbritannien haben der höhere Versorgungsbedarf durch die zunehmende Anzahl an Kindern, die eine Krebserkrankung überleben, der steigende Kostendruck im Gesundheitswesen und der zunehmende Personalmangel bei Pflegefachkräften und Ärzten dazu geführt, dass sich die Rolle der APN schon seit Längerem entwickelt und etabliert hat. ${ }^{3}$

Gemäß der Definition des International Council of Nurses (ICN) kann der Begriff APN wie folgt definiert werden: „Eine Advanced Practice Nurse ist eine registrierte Pflegefachperson, welche sich Expertenwissen, Fähigkeiten zur Entscheidungsfindung bei komplexen Sachverhalten und klinische Kompetenzen für eine erweiterte pflegerische Praxis angeeignet hat. Die Charakteristik der Kompetenzen wird vom Kontext und/oder den Bedingungen des jeweiligen Landes geprägt, in dem sie für die Ausübung ihrer Tätigkeit zugelassen ist. Ein Masterabschluss (Master in Nursing Science) gilt als Voraussetzung." 4,5

Neben dem direkten Zugang zur klinischen Praxis als zentrale Kompetenz gehören Beratung, Expertencoaching, fachliche Führungsaufgaben, ethische Entscheidungsfindung, Mitarbeit an klinischer Forschung und die interdisziplinäre Zusammenarbeit zu den Kernkompetenzen der APN. ${ }^{6}$ Unter dem Schirmkonzept „Advanced Practice Nursing" lassen sich die verschiedenen Rollen der APNs subsummieren: Die CNS hat einen organisations-, edukations- und forschungsbezogenen Schwerpunkt in ihrer Rollenausübung. Im Vordergrund stehen neben der eigenen klinischen Praxis die Anleitung von Pflegefachpersonen und die Entwicklung des Versorgungssystems. Die NP übt vorwiegend eine klinische Tätigkeit an Patienten aus und übernimmt neben pflegerischen Aufgaben auch Tätigkeiten aus dem ärztlichen Bereich. ${ }^{5}$ Die unterschiedlichen Rollen können auf einem Kontinuum dargestellt werden $(\rightarrow$ Abb. 1). In Großbritannien ist für die Ausübung der Rolle der NP oder Advanced Nurse Practitioner ein spezifisches Masterstudium mit einem starken klinischen Fokus Voraussetzung. Dieses Berufsbild hat sich

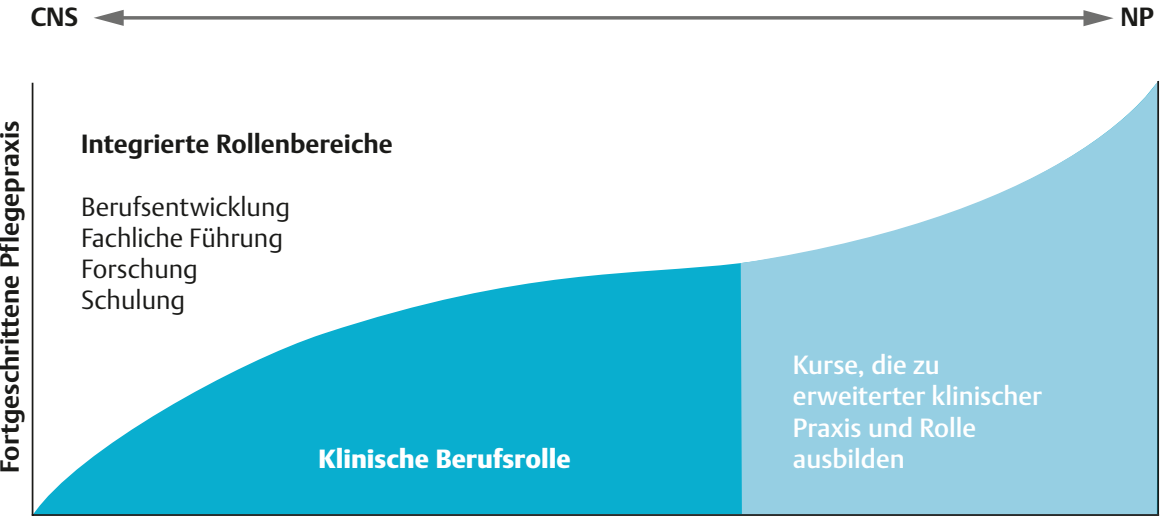

Abb. 1 Kontinuum der APN-Rollen (Übersetzung von Prof. Romy Mahrer-Imhof in Anlehnung an BryantLukosius D (2004 \& 2008). The continuum of advanced practice nursing roles. Unpublished document) ${ }^{7}$.

in Großbritannien in den letzten zwanzig Jahren mit der Ausweitung der klassischen Pflegerollen und damit steigender Autonomie in klinischen Entscheidungsfindungen, der Übernahme von professioneller Verantwortung und der Entwicklung der Pflegeforschung zunehmend etabliert. ${ }^{8}$

In der Schweiz sind derzeit mehr APNs in der Rolle der CNS eingesetzt. Im Schweizer Gesundheitswesen wird das Berufsbild der NP zunehmend diskutiert und durch politische Initiativen vorangetrieben - mit dem Ziel der Ausweitung pflegerischer Verantwortung. ${ }^{5}$

\section{Herausforderungen für Familien mit einem an Krebs erkrankten Kind}

Schätzungen zufolge erkranken weltweit jährlich 175.300 Kinder an Krebs. Zu den häufigsten kindlichen Krebserkrankungen gehören Leukämien, Tumore des zentralen Nervensystems, Lymphome und andere, seltenere Tumore. Dank besserer Therapiemöglichkeiten, je nach Krebsart und kulturellem Kontext, überleben etwa $80 \%$ der Betroffenen. ${ }^{9}$

Für die betroffenen Familien ist eine Krebserkrankung ein einschneidendes und belastendes Lebensereignis. Nach einer Phase des Schocks müssen sie sich neu orientieren. ${ }^{10,11} \mathrm{Ihr}$ Bedürfnis nach Informationen, edukativer Unterstützung und Handlungsanweisungen von professionellen Fachpersonen ist groß. Die Anfangszeit einer Krebserkrankung ist geprägt von Unsicherheit und Ängsten, und die Familien müssen lernen, Vertrauen in die eigenen und in die Fähigkeiten des Fachpersonals aufzubauen. ${ }^{11}$
Die Krebstherapie ist verbunden mit unangenehmen Nebenwirkungen für das Kind wie Übelkeit, Schmerzen oder Beschwerden bei der Stuhlausscheidung. Zudem muss es täglich Medikamente schlucken und hat möglicherweise Angst vor Blutentnahmen und anderen Eingriffen.

Ein Kindergarten- oder Schulbesuch und Freizeitaktivitäten sind phasenweise nur bedingt oder gar nicht möglich und isolieren das Kind von Gleichaltrigen. Die vielen Spitalaufenthalte bedeuten zudem eine Trennung von der Familie. ${ }^{12}$

Geschwisterkinder erhalten weniger Aufmerksamkeit als das kranke Kind, was zu einem Gefühl des Ausgeschlossenseins und Schuldgefühlen führen kann. ${ }^{11,14}$

Die Eltern sind mit der schwierigen Situation konfrontiert, einerseits die Therapie zu unterstützen, haben aber andererseits das Bedürfnis ihr Kind zu schützen und Normalität aufrechtzuerhalten. ${ }^{11,13}$ Nicht zu unterschätzen sind die Herausforderungen, die sich durch die Übernahme der Pflege im häuslichen Umfeld ergeben. Infektionen sind unter der immunsuppressiven Chemo- oder Strahlentherapie lebensbedrohlich für krebskranke Kinder und erfordern eine gute Prävention und zuverlässige Früherkennung durch die Eltern. ${ }^{11}$

Wenn ein Familienmitglied erkrankt, betrifft dies nicht nur das Kind und die Eltern, sondern stets die ganze Familie. Diese Tatsache verpflichtet Pflegefachpersonen, die Familien in die Pflege einzubeziehen. ${ }^{12}$ Familienzentrierte Pflege basiert auf einem Familien-Assessment, Familien-Interventionen und einer beziehungsorientier- 


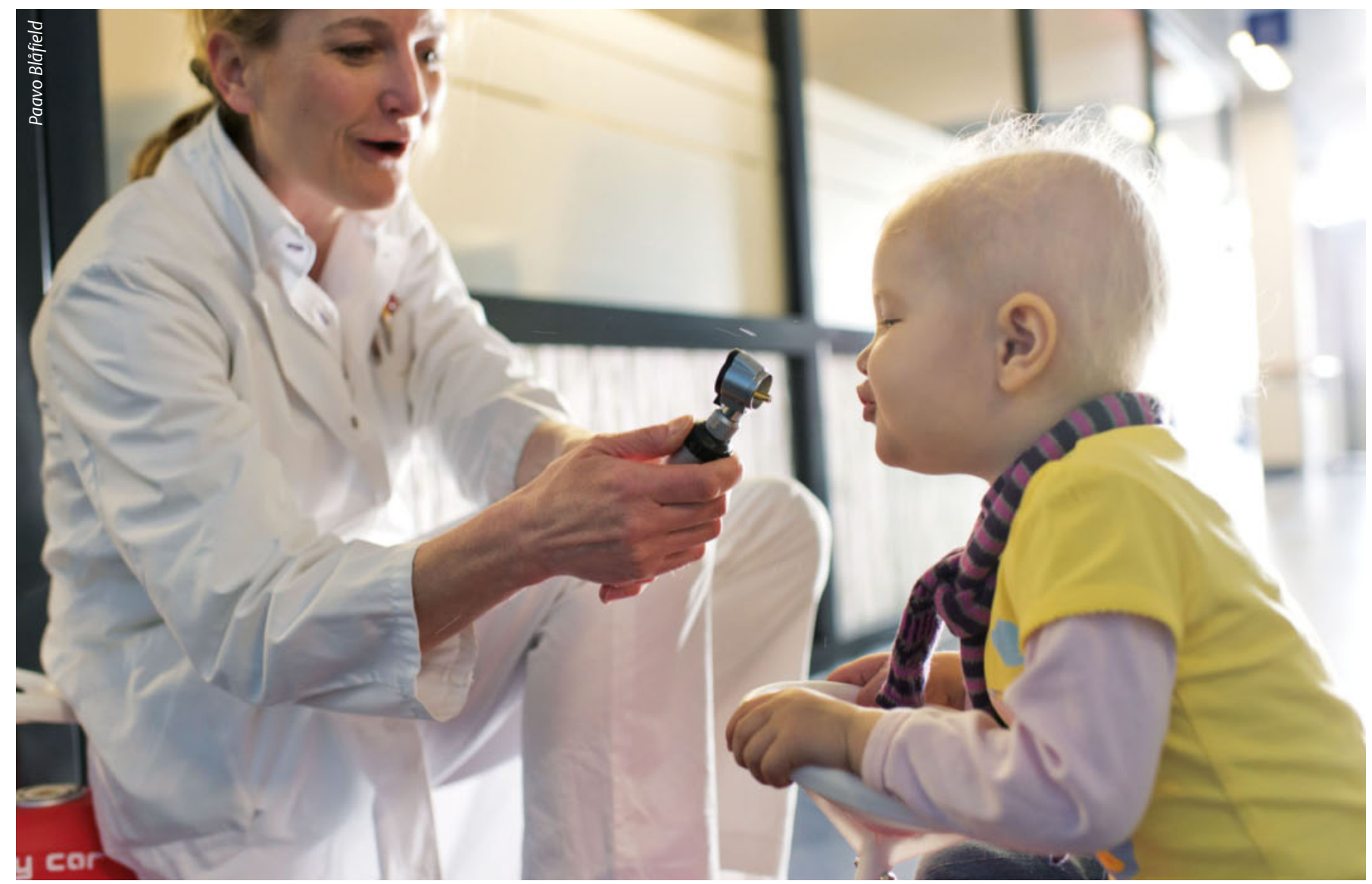

ten Zusammenarbeit mit der Familie. ${ }^{12}$ Von großer Bedeutung sind daher die Unterstützung familiärer Kompetenzen, das Aufzeigen von Lernerfolgen durch das Behandlungsteam und Betreuungskontinuität. ${ }^{11,13}$

Die meisten Krebstherapien sind aggressiv und können bei ehemals krebskranken Kindern zu chronischen Gesundheitsproblemen, Unfruchtbarkeit oder dem Auftreten von Zweittumoren führen, was ein langfristiges und regelmäßiges Gesundheits-Monitoring nach Beendigung der Therapie notwendig macht. ${ }^{15}$

\section{Praxisalltag einer CNS und einer NP in der Kinder-Onkologie und -Hämatologie am Great Ormond Street Hospital}

Beim 6-jährigen Noah (Name geändert) wurde kürzlich eine Leukämie diagnostiziert. In Begleitung seiner Eltern ist er auf einer der zwei stationären onkologischen Abteilungen des Great Ormond Street Hospitals untergebracht. Die Familie wird heute von Amy, einer CNS des APN-Teams, besucht. Anhand der dokumentierten Kran- kengeschichte, der Informationen aus dem interdisziplinären Hämatologie-Meeting und eines Austauschs mit der betreuenden Pflegefachperson informiert sie sich über Noahs Situation. Als CNS ist sie dafür verantwortlich, dass die Familie vor dem Austritt die notwendigen Informationen zur Krankheit und Therapie erhält und ihren Alltag bewältigen kann. Es gilt sicherzustellen, dass die Medikamente korrekt eingenommen werden können und dass die Familie bei Fieber oder einer Verschlechterung des Allgemeinzustands von Noah richtig reagiert.

Amy bespricht mit Noahs Eltern den ersten Chemotherapieblock und die diagnostischen Interventionen der kommenden Wochen. Einfachere Behandlungen und Chemotherapien werden in Noahs lokalem Spital durchgeführt, die restliche Therapie findet am Great Ormond Street Hospital statt. Vor dem Gespräch hatte Amy bereits mit der CNS des lokalen Spitals telefonisch die Termine koordiniert.

Für das Familiengespräch nimmt sich Amy Zeit und geht auf Fragen und Sorgen der Eltern ein. Sie eruiert den Wissensstand und wiederholt wo nötig verschiedene Informationen. Danach vereinbart sie mit der Familie ein weiteres Gespräch, um über die Vor- und Nachteile diverser zentralvenöser Zugänge zu informieren und der Familie die Entscheidung zu erleichtern.

An diesem Tag besucht Amy noch weitere Familien für Edukationsgespräche. Zwischendurch koordiniert sie Termine mit lokalen Spitälern und aktualisiert die Chemotherapie-Protokolle in den Patientenakten.

Das APN-Team hat eine E-Mail-Adresse, die von allen CNS und NP benutzt wird. Das ermöglicht die effiziente Bearbeitung der Fragen und Anliegen von Familien oder Fachpersonal und gewährleistet zugleich den Informationsfluss im APN-Team. Amy kontaktiert eine Mutter, nachdem diese sich via E-Mail wegen einer Mundschleimhautentzündung ihrer Tochter gemeldet hatte. Eine kurze E-Mail-Notiz informiert die NP und CNS der ambulanten Tagesklinik über die vereinbarten Maßnahmen. 
Am darauffolgenden Tag findet die ärztliche Visite statt, an der mindestens eine CNS oder NP teilnimmt. Amy ergänzt die medizinischen Informationen mit Informationen aus den Gesprächen und Schulungen mit den Familien und fordert wo nötig Unterstützung durch die zuständigen Ärzte oder Sozialarbeitenden ein. Die betreuenden Pflegefachpersonen nehmen an der ärztlichen Visite nicht teil. Die APN muss sich daher gut über die Familien informieren.

Im Anschluss schaut Amy zusammen mit einer CNS-Dermatologie bei einem leukämiekranken Kleinkind mit ausgeprägter Windeldermatitis vorbei. Die CNSDermatologie erfasst den Hautstatus des Kindes, diagnostiziert eine Candida-Infektion und macht dem Behandlungsteam und der Mutter einen Therapievorschlag.

Nach einem weiteren Informationsgespräch mit Noahs Familie entscheidet diese sich für eine Port-à-Cath-Implantation. Amy kümmert sich im Anschluss um einen Operationstermin auf der ambulanten Tagesklinik.

Ein erheblicher Teil der Krebstherapie findet nach einer ersten stationären Zeit ambulant statt. Auf der onkologischen Tagesklinik des Great Ormond Street Hospitals arbeiten eine CNS und zwei NP des APN-Teams. NP Mary leitet das APN-Team, arbeitet aber auch eng mit dem ärztlichen Dienst zusammen: Sie führt körperliche Untersuchungen der Kinder vor der Chemotherapie oder Eingriffen unter Vollnarkose durch und beurteilt ihre Laborwerte. Sofern die Kinder alle Kriterien erfüllen, wird die Chemotherapie durch die betreuende Pflegefachperson auf der Tagesklinik verabreicht. Mary klärt die Familien vor Lumbal- und Knochenmarkspunktionen über die Notwendigkeit und die Risiken des Eingriffs auf und holt ihre schriftliche Einwilligung ein. Da NP in England die Kompetenz zur Verschreibung von Medikamenten haben, muss Mary die Dosierung der oralen Chemotherapie laufend anpassen und weitere Medikamente wie Antiemetika verschreiben.

Noah kommt nach der Entlassung von der stationären Abteilung und einer Woche daheim zum ersten Mal in die Tagesklinik. Mary untersucht Herz und Lunge von Noah, kontrolliert seine Refle- xe und beurteilt die Hautverhältnisse. Sie fragt die Familie nach Infektionen und Beschwerden in der vergangenen Woche und überprüft im Anschluss die Laborwerte des Bluts. Noah erfüllt alle Kriterien für die Knochenmarkspunktion und die Chemotherapie. Mary schreibt einen Bericht zum Untersuchungsbefund und zum weiteren Prozedere, der auch an das Behandlungsteam des lokalen Spitals von Noah weitergeleitet wird.

\section{Persönlicher Eindruck der Arbeits- felder der CNS und NP}

Ich erlebte die Begleitung und Pflege der Familien krebskranker Kinder am Great Ormond Street Hospital durch das APNTeam als sehr beziehungsorientiert. Die APNs nehmen ihre Rolle als Bezugspersonen wahr und stehen über die gesamte Krankheitsdauer via E-Mail, telefonisch und vor Ort in Kontakt mit den Familien. Sie begleiten und unterstützen die Pflegefachpersonen bei der Edukation, Austrittsplanung und in komplexen sozialen oder pflegerischen Situationen. Sowohl NP als CNS nahm ich, bedingt durch ihre Expertise und Ausbildung, als wichtige Ansprechpartner der Pflegefachpersonen wahr.

Die Tatsache, dass das Austrittsmanagement und die Edukation durch die APN übernommen werden, war mir aus vereinzelten Institutionen in der Schweiz bekannt und macht in Anbetracht mangelnder Kontinuität und knapper Zeitressourcen des Pflegefachpersonals im Schichtbetrieb durchaus Sinn.

Die hohe Kontinuität in der Begleitung der Familien geht zudem über die akute Krankheitsphase hinaus: Die „Survivors“, also Kinder, die die Krebserkrankung überleben, werden durch eine NP in einer pflegegeleiteten Sprechstunde auf mögliche Spätfolgen über Jahre betreut und in der Übertritts-Phase in die Erwachsenenonkologie begleitet. Nach meinen Erfahrungen existiert dieses pflegegeleitete Angebot derzeit in der Schweizer Kinderonkologie nicht und wäre für die Zukunft erstrebenswert.

Obwohl die Edukation der Familien durch die CNS auf der Basis eines Familieninformations-Ordners erfolgte, erlebte ich diese als individuell angepasst: Sprachliche Barrieren wurden ebenso wie der emotionale Zustand der Betroffenen berücksichtigt. Bei Bedarf wurden mehrere Gespräche täglich bei einer Familie geführt. Es wurde deutlich, dass dabei die Zeitressourcen des APN-Teams größer waren als jene der Pflegefachpersonen im Schichtbetrieb, was letztendlich den Familien zugute kam.

In Beratungssituationen nahm ich regelmäßig wahr, wie die APNs Wertschätzung und Anerkennung zum Ausdruck brachten, was als wichtige Intervention in der Familienzentrierten Pflege gilt. ${ }^{11}$ Familienzentrierte Pflege ist den APN und Pflegefachpersonen am Great Ormond Street Hospital ein Begriff und wird in der Ausbildung teilweise gelehrt. Ähnlich wie in meinem Berufsfeld werden Elemente der Familienzentrierten Pflege berücksichtigt. Sie ist jedoch am Great Ormond Street Hospital nicht anhand eines Modells oder Konzepts offiziell integriert.

Die Trennung der Aufgabenbereiche zwischen CNS und NP konnte ich deutlich wahrnehmen. Die NP führte beispielsweise keine Edukationsgespräche mit den Familien durch. Sie tauschte sich im Anschluss an die Untersuchung eines Kindes mit der CNS aus und delegierte falls nötig die Edukationsgespräche weiter. Die CNS wiederum führt keine körperlichen Untersuchungen durch, ist nicht verschreibungsberechtigt und stellt keine Diagnosen. Obwohl die Tätigkeit der NP im Gegensatz zur Rolle einer CNS an die Rolle einer Ärztin erinnert, zeigte die Art und Weise, wie Mary die Kinder spielerisch untersuchte, mit ihnen kommunizierte und dabei Kinderlieder sang, dass sie Pflegefachperson ist. Dabei wirkte sie auf mich in der Ausführung der körperlichen Untersuchung und in ihrer Urteilsfähigkeit sehr kompetent und erfahren.

Das APN-Team am Great Ormond Street Hospital hat eine entscheidende Rolle in der interdisziplinären Zusammenarbeit eingenommen. Das Team bezeichnet sich als „Keyworkers“, also Schlüssel- oder Bezugspersonen. Diese Schlüsselfunktion kam für mich insbesondere in der interdisziplinären Zusammenarbeit zum Ausdruck. Die APNs koordinieren viele Termine im Rahmen der Krebstherapie: Sie organisieren nach Studienprotokoll die Termine für die Chemotherapie, damit 
verbundene diagnostische Untersuchungen und koordinieren die Zusammenarbeit mit den lokalen Spitälern. Ich war positiv überrascht, wie selbstständig und eigenverantwortlich die APNs in den unterschiedlichen Rollen den Behandlungsprozess organisieren. Die Aufgabengebiete, insbesondere der NP und der Ärzte, überschneiden sich. Dabei entstand entgegen meinen Erwartungen nicht der Eindruck, dass sie in Konkurrenz zueinander stehen. Ärzte und APNs profitieren von der $\mathrm{Zu}$ sammenarbeit und begegnen sich auf Augenhöhe.

\section{FAZIT}

Im Rückblick lässt sich zusammenfassen, dass sich APNs in Großbritannien in einer Schlüsselfunktion für die Pflege und in der Begleitung von Familien krebskranker Kinder befinden. Dadurch können Kontinuität und eine beziehungsorientierte Pflege für die Familien in einem multidisziplinären Setting gewährleistet werden. Obwohl sich die Gesundheitssysteme in Großbritannien und der Schweiz unterscheiden, sind APNs mittlerweile auch in der Schweiz ein Mehrwert für die Versorgung krebskranker Kinder oder Kinder mit anderen chronischen Erkrankungen. Die APN trägt mit ihrem fachspezifischen Wissen im multidisziplinären Team dazu bei, eine qualitativ gute Therapie, Pflege und Begleitung der Familien zu gewährleisten.

\section{$\rightarrow$ LITERATUR}

1 Great Ormond Street Hospital for Children NHS Foundation Trust. About us. 2015. Online unter www.gosh.nhs.uk/about-us, letzter Zugriff am 15.09.2015

2 Great Ormond Street Hospital for Children NHS Foundation Trust. Haematology and oncology information for parents and visitors. 2015. Online unter www.gosh.nhs.uk/medicalinformation/clinical-specialties/haematologyand-oncology-information-parents-andvisitors, letzter Zugriff am 15.09.2015

3 De Geest S et al. Introducing advanced practice nurses/nurse practitioners in health care systems: a framework for reflection and analysis. Swiss Medical Weekly 2008, 138(43-44): 621-628

4 International Council of Nurses. Definition and characteristics of the nurse practitioner and advanced practice roles. 2001. Online unter http:// international.aanp.org/Practice/APNRoles, letzter Zugriff am 15.09.2015
5 Mahrer Imhof $R$ et al. Expertenbericht APN: Expertengruppe Schweizerischer Verein für Pflegewissenschaft (VFP). 2012. Online unter www.pflegeforschung-vfp.ch/download/58/ page/23761_dl_2012-04-03_vfp-apnexpertenbericht_final_d.pdf, letzter Zugriff am 23.08.2015

6 Hamric $A B$ et al. Advanced practice nursing: an integrative approach. 5. ed.. St. Louis, Missouri: Elsevier/Saunders; 2014

7 DiCenso, A, Bryant-Lukosius, D. Clinical Nurse Specialists and Nurse Practitioners in Canada: A decision support synthesis. Ottawa: Canadian Health Services Research Foundation; 2010

8 Royal College of Nursing. Specialist and advanced children's and young people's nursing practice in contemporary health care: guidance for nurses and commissioners. 2014, 1-31. Online unterwww.rcn.org.uk/_data/assets/pdf_ file/0009/580725/004579.pdf, letzter Zugriff am 23.08.2015

9 American Cancer Society. Global Cancer Facts E Figures. 2nd ed. American Cancer Society: Atlanta; 2011. Online unter www.cancer.org/acs/ groups/content/@epidemiologysurveilance/ documents/document/acspc-027766.pdf, letzter Zugriff am 20.09.2015

10 Schweitzer R, Griffiths M, Yates P. Parental experience of childhood cancer using Interpretative Phenomenological Analysis. Psychology \& Health 2012, 27(1476-8321 (Electronic)): 704-720

11 Tiesmeyer K. Familien mit einem krebskranken Kind: Möglichkeiten und Grenzen edukativer Unterstützung. 1. ed. Bern: Hans Huber; 2012

12 Wright LM, Leahey M. Familienzentrierte Pflege: Lehrbuch für Familien-Assessment und Interventionen. 2., vollst. überarb., erg. Aufl. Programmbereich Pflege. Bern: Hans Huber; 2014

13 Darcy L et al. The Everyday Life of the Young Child Shortly After Receiving a Cancer Diagnosis, From Both Children's and Parent's Perspectives. Cancer Nursing 2014, 37(6): 445-456

14 Prchal A, Landolt MA. How siblings of pediatric cancer patients experience the first time after diagnosis: a qualitative study. Cancer Nurs 2012, 35(2): 133-40

15 Kuehni C. Factsheet Schweizer Kinderkrebsregister. Schweizer Kinderkrebsregister, 2015: 1-2. Online unter www.kinderkrebsregister.ch/ fileadmin/KKR08/uploads/pdf/Factsheet/ 20150512_Factsheet_SKKR.pdf, letzer Zugriff am 30.08.2015

\section{$\rightarrow$ DANK}

Ich bedanke mich herzlich beim ganzen Pflegeteam der Onkologie, Hämatologie und Hämophilie am Great Ormond Street Hospital für Kinder. Zu großem Dank bin ich insbesondere Frau Emily Baker, Lead Nurse for Education ICI-LM, für die reibungslose und engagierte Organisation des Work-Shadowings verpflichtet.

\section{E AUTORINNEN}

Seraina Brooks, Pflegefachfrau, $\mathrm{BSCN}$, cand. MScN

Arbeitet seit neun Jahren in der Akutpädiatrie, unter anderem in der Pflege onkologischer und hämatologischer Patienten im Alter von 0-18 Jahren. Ihr spezielles Interesse gilt Familien mit chronisch kranken Kindern und der Pflege von Kindern und Familien mit Migrationshintergrund. Für ihre Bachelorarbeit hat sie eine Literaturrecherche zum Thema „Kriegstraumatisierungen bei Kindern und Jugendlichen “ durchgeführt und daraus Erkenntnisse für die Pflege dieser Population gewonnen. Als Masterarbeit wird sie eine phänomenologische Studie zur Kommunikation zwischen hörbeeinträchtigten Kindern und Pflegefachpersonen im Spital durchführen.

E-Mail: seraina.brooks@bluewin.ch

\section{Dr. rer. medic}

Daniela Händler-Schuster Dozierende im Studiengang Master of Science in Pflege und Projektleiterin im Bereich Forschung und Entwicklung Pflege an der Zürcher Hochschule für Angewandte Wissenschaften (ZHAW).

Schwerpunkt Ihrer Forschung ist u. a. das Leben mit Beeinträchtigungen in unterschiedlichen Lebensspannen sowie die Professionalisierung der Pflege. E-Mail: haed@zhaw.ch

Prof. Dr. Romy Mahrer-Imhof Leitet den Studiengang Master of Science in Pflege an der Zürcher Hochschule für Angewandte Wissenschaften (ZHAW). Sie setzt sich seit Jahren für APN ein und unterrichtet $z u$ Rollenentwicklung der Advanced Practice

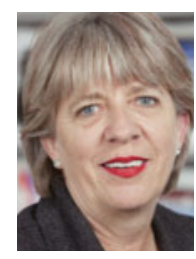
Nurses in verschiedenen Studiengängen. Sie ist Professorin für familienzentrierte Pflege und leitet Forschungsprojekte, die die Auswirkungen von Erkrankung und Beeinträchtigungen auf das familiale System untersuchen.

E-Mail: mahr@zhaw.ch

\section{BIBLIOGRAFIE}

DOI 10.1055/s-0042-101615

JuKiP 2016; 5: 57-61

(c) Georg Thieme Verlag KG

Stuttgart · New York · ISSN 1439-2569 